\title{
Supplementary Material: Bayes Linear Analysis of Risks in Sequential Optimal Design Problems
}

\author{
Matthew Jones and Michael Goldstein \\ Department of Mathematical Sciences, Durham University \\ Lower Mountjoy, Stockton Road, Durham, UK, DH1 3LE \\ e-mail: m.j.jones@durham.ac.uk; michael.goldstein@durham.ac.uk \\ Philip Jonathan \\ Department of Mathematics and Statistics, Lancaster University, \\ LA1 $4 Y W$, United Kingdom \\ Shell Research Limited, London SE1 7NA, UK \\ e-mail: philip.jonathan@shell.com \\ David Randell \\ Shell Global Solutions International BV, Grasweg 31, \\ $1031 \mathrm{HW}$ Amsterdam, NL \\ e-mail: david.randell@shell.com
}

\section{Contents}

S1 Bayes Linear Analysis . . . . . . . . . . . . . . . . . . . . . 2

S1.1 General Bayes Linear Analysis _. . . . . . . . . . . . . 3

S1.2 Second-order Emulation . . . . . . . . . . . . . . . 3

S1.2.1 Adjustment . . . . . . . . . . . . . . . . . 4

S1.2.2 Fixing the Correlation Parameters . . . . . . . . . 5

S1.3 Bayes Linear Quadrature . . . . . . . . . . . . . . . . . 6

S1.4 Bayes Linear Uncertainty Analysis . . . . . . . . . . . . . 7

S2 Design Algorithm . . . . . . . . . . . . . . . . . . 8

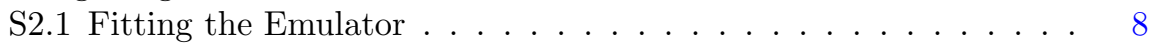

S2.1.1 Evaluating the Risk . . . . . . . . . . . . 8

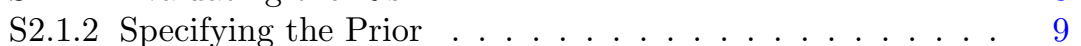

S2.1.3 Jointly Updating . . . . . . . . . . . . . . . . . . . 9

S2.1.4 Checking Inter-Wave Correspondence . . . . . . . . . . 10

S2.2 Computed the Expected Risk . . . . . . . . . . . . . . 10

S2.3 Characterising the Optimal Design Space . . . . . . . . . . 10

S2.4 Designing for Future Waves . . . . . . . . . . . . . . . 12

S3 Example: Linear Model . . . . . . . . . . . . . . . . . . . . . . . 12

S3.1 First Wave: Details . . . . . . . . . . . . . . . . . . . 12

S3.1.1 Stage 2: Emulating the risk . . . . . . . . . . 12 
S3.1.2 Stage 2: Computing the expected risk . . . . . . . . . 13

S3.1.3 Stage 2: Characterising the candidate design space . . . . 13

S3.1.4 Stage 1 . . . . . . . . . . . . . . . . 14

S3.2 Second Wave: Details . . . . . . . . . . . . . . . . . . 14

S4 Example: Airborne Sensing Problem . . . . . . . . . . . . . . . . . 14

S4.1 Prior Specification and Posterior Risk . . . . . . . . . . . . 15

S4.2 Emulator Fit: Details . . . . . . . . . . . . . . . . . . 15

A Squared Exponential Correlation Function . . . . . . . . . . . . 16

A.1 Integrals: Gaussian Case . . . . . . . . . . . . . . . . . 17

A.2 Integrals: Uniform Case . . . . . . . . . . . . . . . . 17

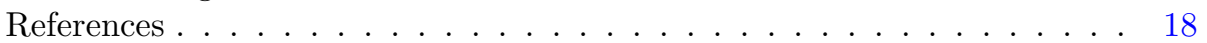

\section{S1. Bayes Linear Analysis}

A Bayes linear analysis is a framework within which we update our prior beliefs about a collection of quantities in the light of observations; it differs from a probabilistic Bayesian analysis in that it treats expectation rather than probability as a primitive. Prior expectations, variances and covariances are specified for all components of the problem, and then upon learning the values of certain quantities, beliefs about the remainder are updated using linear fitting. A comprehensive introduction to the Bayes linear framework is provided in Goldstein and Wooff (2007).

The Bayes linear approach can be viewed either as a generalization of the fully Bayesian approach in which we remove the requirement that we must make a full probability specification for all quantities; alternatively, it can be viewed as a simple approximation to the fully Bayesian treatment, for situations where we are unwilling to make a full probability specification for all quantities, or where the full Bayesian analysis would be too difficult or time-consuming to be practical. Regardless of our views on the general appropriateness of a Bayes linear treatment, the simplicity of the Bayes linear framework allows us to make progress in problems where this would not be possible using a fully probabilistic specification; specifically, the use of Bayes linear methods can introduce tractability into design problems which would otherwise be too computationally difficult to solve.

In this section, we outline the Bayes linear methods which are used for analysis in the main article. In Section S1.1, we introduce the elements of a general Bayes linear analysis; then, in Section S1.2, we use these elements to develop a common model for an uncertain function. In Section S1.3, we demonstrate how this type of model can be used to compute our beliefs about the expectation of a function, and in Section S1.4, we outline how it can be used to approximate a general, complex model in a way that makes the approximate procedure from Section 3 of the main article tractable. 


\section{S1.1. General Bayes Linear Analysis}

Suppose that we are interested in a collection of quantities $C=\{A, B, D\}$, where $A=\left\{A_{1}, \ldots, A_{n_{A}}\right\}, B=\left\{B_{1}, \ldots, B_{n_{B}}\right\}$ and $D=\left\{D_{1}, \ldots, D_{n_{D}}\right\}$, and that we can make a second-order prior specification for the collection, consisting of expectations and variances for all components and covariances between all pairs of components. If we then observe the values of the collection $D$, we want to know how we should alter our beliefs about the other quantities in the light of this information. Under the Bayes linear framework, we use the new information to compute adjusted beliefs (Goldstein and Wooff, 2007).

Definition S1.1. Our adjusted expectation for the collection B given observation of the collection $D$ is

$$
E_{D}[B]=E[B]+\operatorname{Cov}[B, D] \operatorname{Var}[D]^{-1}[D-E[D]]
$$

Definition S1.2. Our adjusted variance for the collection $B$ given observation of the collection $D$ is

$$
\operatorname{Var}_{D}[B]=\operatorname{Var}[B]-\operatorname{Cov}[B, D] \operatorname{Var}[D]^{-1} \operatorname{Cov}[D, B]
$$

Definition S1.3. Our adjusted covariance between the collections $B$ and $A$ given observation of the collection $D$ is

$$
\operatorname{Cov}_{D}[A, B]=\operatorname{Cov}[A, B]-\operatorname{Cov}[A, D] \operatorname{Var}[D]^{-1} \operatorname{Cov}[D, B]
$$

\section{S1.2. Second-order Emulation}

A second-order emulator is a particular Bayes linear model which we use to predict the behaviour of a function over its whole input domain based on a set of assumptions about its structure and a number of observations made (possibly with error) at known input locations. Emulation of complex functions can also be carried out using either a fully probabilistic specification (see, for example, Kennedy and O'Hagan (2001) or Goldstein and Rougier (2004)). We adopt the Bayes linear approach because it eliminates the need for us to make highorder moment specifications that we do not necessarily believe (as discussed in Goldstein and Wooff (2007)), and because it simplifies some of the analyses that we may wish to perform using our fitted emulator (e.g. learning about uncertain inputs; see Goldstein and Rougier (2006)).

Using $f(\theta)=\left\{f_{1}(\theta), \ldots, f_{n_{f}}(\theta)\right\}$ to denote a general multi-output function of inputs $\theta=\left\{\theta_{1}, \ldots, \theta_{n_{\theta}}\right\}$, our model consists of a regression surface and a residual component

$$
f_{i}(\theta)=\sum_{p} \beta_{i p} g_{p}(\theta)+u_{i}(\theta)
$$

where $g=\left\{g_{1}, \ldots, g_{n_{g}}\right\}$ is a set of known basis functions, the $\beta_{i p}$ are corresponding uncertain weights, and $u_{i}$ is a correlated residual process. We make a secondorder prior specification for each of the uncertain components of the model, 
which consists of expectations $\mathrm{E}\left[\beta_{i p}\right]$ for all weights, covariances $\operatorname{Cov}\left[\beta_{i p}, \beta_{j q}\right]$ between pairs of weights and a covariance function $\operatorname{Cov}\left[u_{i}(\theta), u_{j}\left(\theta^{\prime}\right)\right]$ for the residual process. This prior specification is then combined with a set of noisy observations of the function to produce adjusted expectations $\mathrm{E}_{F}\left[f_{i}(\theta)\right]$ for all new inputs $\theta$ and adjusted covariances $\operatorname{Cov}_{F}\left[f_{i}(\theta), f_{j}(\theta)\right]$ for all new pairs of inputs. This adjustment procedure is detailed in Section S1.2.1, and the determination of the correlation parameters for the covariance function is considered in Section S1.2.2.

This type of model is useful in a number of different settings. If $f$ is a complex function with high-dimensional input and output spaces that takes a long time to evaluate on a computer, then a model of the form (S4) can be fitted as a surrogate representation. A carefully specified model can be used to capture the important features of the function's behaviour, and can be run in a fraction of the time that it would take to run the function itself; the surrogate model can therefore be used to carry out more detailed analyses which would not originally have been possible.

Throughout the remainder of this section, we assume the Einstein summation convention, under which repeated indices are summed over; for example $\sum_{p} \beta_{i p} g_{p}(\theta)=\beta_{i p} g_{p}(\theta)$.

\section{S1.2.1. Adjustment}

Suppose that we observe the noise-corrupted function output at $n_{F}$ known input locations $\left\{\theta_{1}, \ldots, \theta_{n_{F}}\right\}$. We use $F_{i k}$ to denote the $i^{\text {th }}$ component of the $k^{\text {th }}$ function output, and we assume that

$$
F_{i k}=f_{i}\left(\theta_{k}\right)+\eta_{i k}
$$

If we assume that the noise components have mean zero, and we specify $\operatorname{Cov}\left[\eta_{i k}, \eta_{j l}\right]$ for all pairs of noise terms, then combining this with our prior specification from earlier, we have that

$$
\begin{aligned}
\mathrm{E}\left[F_{i k}\right] & =\mathrm{E}\left[\beta_{i p}\right] G_{p k} \\
\operatorname{Cov}\left[F_{i k}, F_{j l}\right] & =G_{p k} \operatorname{Cov}\left[\beta_{i p}, \beta_{j q}\right] G_{q l}+\operatorname{Cov}\left[u_{i}\left(\theta_{k}\right), u_{j}\left(\theta_{l}\right)\right]+\operatorname{Cov}\left[\eta_{i k}, \eta_{j l}\right]
\end{aligned}
$$

where the $G_{p k}=g_{p}\left(\theta_{k}\right)$ are the elements of the usual basis matrix. Our adjusted beliefs about the function outputs at new input settings are now

$$
\begin{aligned}
\mathrm{E}_{F}\left[f_{i}(\theta)\right]= & \mathrm{E}_{F}\left[\beta_{i p}\right] g_{p}(\theta)+\mathrm{E}_{F}\left[u_{i}(\theta)\right] \\
\operatorname{Cov}_{F}\left[f_{i}(\theta), f_{j}\left(\theta^{\prime}\right)\right]= & g_{p}(\theta) \operatorname{Cov}_{F}\left[\beta_{i p}, \beta_{j q}\right] g_{q}\left(\theta^{\prime}\right)+g_{p}(\theta) \operatorname{Cov}_{F}\left[\beta_{i p}, u_{j}\left(\theta^{\prime}\right)\right] \\
& +\operatorname{Cov}_{F}\left[u_{i}(\theta), \beta_{j q}\right] g_{q}(\theta)+\operatorname{Cov}\left[u_{i}(\theta), u_{j}\left(\theta^{\prime}\right)\right] \quad(\mathrm{S} 6)
\end{aligned}
$$

where the individual adjusted components are detailed below.

Adjusted expectation The components of the adjusted expectation are computed through simple application of the Bayes linear adjustment rule (S1). For 
the regression coefficients, we have

$$
\mathrm{E}_{F}\left[\beta_{i p}\right]=\mathrm{E}\left[\beta_{i p}\right]+\operatorname{Cov}\left[\beta_{i p}, F_{r s}\right] \operatorname{Var}[F]_{r s t u}^{-1}\left[F_{t u}-\mathrm{E}\left[F_{t u}\right]\right]
$$

where

$$
\operatorname{Cov}\left[\beta_{i p}, F_{r s}\right]=\operatorname{Cov}\left[\beta_{i p}, \beta_{r t}\right] G_{t s}
$$

and $\operatorname{Var}[F]^{-1}$ is computed as follows: we define $\tilde{F}$ such that $\tilde{F}_{\left(i+(k-1) n_{f}\right)}=F_{i k}$, and set

$$
\operatorname{Var}[F]_{i k j l}^{-1}=\operatorname{Var}[\tilde{F}] \underset{\left(i+(k-1) n_{f}\right),\left(j+(l-1) n_{f}\right)}{-1}
$$

For the residual process, the adjusted expectation is

$$
\mathrm{E}_{F}\left[u_{i}(\theta)\right]=\operatorname{Cov}\left[u_{i}(\theta), F_{r s}\right] \operatorname{Var}[F]_{r s t u}^{-1}\left[F_{t u}-\mathrm{E}\left[F_{t u}\right]\right]
$$

where

$$
\operatorname{Cov}\left[u_{i}(\theta), F_{r s}\right]=\operatorname{Cov}\left[u_{i}(\theta), u_{r}\left(\theta_{s}\right)\right]
$$

Adjusted covariance Likewise, the components of the adjusted covariance are computed through repeated application of S3. For the regression coefficients

$$
\operatorname{Cov}_{F}\left[\beta_{i p}, \beta_{j q}\right]=\operatorname{Cov}\left[\beta_{i p}, \beta_{j q}\right]-\operatorname{Cov}\left[\beta_{i p}, F_{r s}\right] \operatorname{Var}[F]_{r s t u}^{-1} \operatorname{Cov}\left[F_{t u}, \beta_{j q}\right]
$$

and for the residual process

$$
\begin{aligned}
\operatorname{Cov}_{F}\left[u_{i}(\theta), u_{j}\left(\theta^{\prime}\right)\right]=\operatorname{Cov} & {\left[u_{i}(\theta), u_{j}\left(\theta^{\prime}\right)\right] } \\
& -\operatorname{Cov}\left[u_{i}(\theta), F_{r s}\right] \operatorname{Var}[F]_{r s t u}^{-1} \operatorname{Cov}\left[F_{t u}, u_{j}\left(\theta^{\prime}\right)\right]
\end{aligned}
$$

Covariances between the components are

$$
\operatorname{Cov}_{F}\left[\beta_{i p}, u_{j}\left(\theta^{\prime}\right)\right]=-\operatorname{Cov}\left[\beta_{i p}, F_{r s}\right] \operatorname{Var}[F]_{r s t u}^{-1} \operatorname{Cov}\left[F_{t u}, u_{j}\left(\theta^{\prime}\right)\right]
$$

where we have used the fact that the $\beta_{i p}$ and the $u_{j}$ are a priori uncorrelated.

\section{S1.2.2. Fixing the Correlation Parameters}

Generally, the covariance function which we choose will have the following form

$$
\operatorname{Cov}\left[u_{i}(\theta), u_{j}\left(\theta^{\prime}\right) \mid \lambda\right]=V_{i j} k\left(\theta, \theta^{\prime} \mid \lambda\right)
$$

where $V$ is a matrix of marginal covariances, and $k$ is a correlation function which depends on a set of parameters $\lambda$. In a fully Bayesian analysis, we would specify prior beliefs about $\{V, \lambda\}$ and then update these beliefs using the data $F$; generally, however, this procedure is too computationally intensive to be practical here. Instead, then, we adopt a common approximate treatment (see, for example, Kennedy and O'Hagan (2001), Craig et al. (2001)); we empirically fix $V$ to a setting $\hat{V}$ which is empirically determined from the residuals of the initial mean regression surface $\mathrm{E}\left[\beta_{i p}\right] g_{p}(\theta)$, and we fix the correlation parameters $\lambda$ to a setting $\hat{\lambda}$ determined through leave-one-out cross validation. For an outline of the leave-one-out cross validation procedure, see e.g. Rasmussen and Williams (2006). 


\section{S1.3. Bayes Linear Quadrature}

Suppose that we are interested in the value of the expectation of the function $f$ with respect to a certain subset of its inputs. We split the input set $\theta=\{a, b\}$, where the values of the $b=\left\{b_{1}, \ldots, b_{n_{b}}\right\}$ are known, and the $a=\left\{a_{1}, \ldots, a_{n_{a}}\right\}$ are unknown, with our beliefs about these components specified through a distribution $p(a)$. We want to compute

$$
\bar{f}_{i}(b)=\int f_{i}(a, b) p(a) d a
$$

but we cannot do so directly; this could be because we do not have access to an algebraic expression for $f$, or it could be because we do not have access to an expression for the definite or indefinite integral for this combination of $f$ and $p$. In either case, a simple approximating strategy is to fit a model of the kind outlined in section S1.2 and then to integrate.

This strategy was proposed for Gaussian process models by O'Hagan (1991), who referred to it as 'Bayesian quadrature'; it was also considered by Rasmussen and Ghahramani (2002), who called it 'Bayesian Monte Carlo'. In both cases, the same procedure is followed: a Gaussian process model is fitted to $f$, and then the mean and covariance functions for the expectation $\bar{f}$ of $f$ are computed as the expectations of the mean and covariance functions. We perform the same calculations as these authors, but in the Bayes linear setting.

The (adjusted) expectation of $\bar{f}$ is simply the integral of the adjusted expectation of $f$

$$
\mathrm{E}_{F}\left[\bar{f}_{i}(b)\right]=\int \mathrm{E}_{F}\left[f_{i}(a, b)\right] p(a) d a
$$

The covariance between $\bar{f}$ values at any pair of input points is also just the integral of the covariance between $f$ values

$$
\operatorname{Cov}_{F}\left[\bar{f}_{i}(b), \bar{f}_{j}\left(b^{\prime}\right)\right]=\iint \operatorname{Cov}_{F}\left[f_{i}(a, b), f_{j}\left(a^{\prime}, b^{\prime}\right)\right] p(a) p\left(a^{\prime}\right) d a d a^{\prime}
$$

Substituting the expressions (S5) and (S6) into the expressions (S7) and (S8), we obtain

$$
\begin{aligned}
\mathrm{E}_{F}\left[\bar{f}_{i}(b)\right]= & \mathrm{E}_{F}\left[\beta_{i p}\right] \bar{g}_{p}(b)+\mathrm{E}_{F}\left[\bar{u}_{i}(b)\right] \\
\operatorname{Cov}_{F}\left[\bar{f}_{i}(b), \bar{f}_{j}\left(b^{\prime}\right)\right]= & \bar{g}_{p}(b) \operatorname{Cov}_{F}\left[\beta_{i p}, \beta_{j q}\right] \bar{g}_{q}\left(b^{\prime}\right)+\bar{g}_{p}(b) \operatorname{Cov}_{F}\left[\beta_{i p}, \bar{u}_{j}\left(b^{\prime}\right)\right] \\
& +\operatorname{Cov}_{F}\left[\bar{u}_{i}(b), \beta_{j q}\right] \bar{g}_{q}(b)+\operatorname{Cov}\left[\bar{u}_{i}(b), \bar{u}_{j}\left(b^{\prime}\right)\right]
\end{aligned}
$$

where

$$
\bar{g}_{p}(b)=\int g_{p}(a, b) p(a) d a
$$

and

$$
\begin{aligned}
& \operatorname{Cov}_{F}\left[\bar{u}_{i}(b), \bar{u}_{j}\left(b^{\prime}\right)\right]= \operatorname{Cov}\left[\bar{u}_{i}(b), \bar{u}_{j}\left(b^{\prime}\right)\right] \\
&-\operatorname{Cov}\left[\bar{u}_{i}(b), F_{r s}\right] \operatorname{Var}[F]_{r s t u}^{-1} \operatorname{Cov}\left[F_{t u}, \bar{u}_{j}\left(b^{\prime}\right)\right] \\
& \operatorname{Cov}_{F}\left[\beta_{i p}, \bar{u}_{j}\left(b^{\prime}\right)\right]=-\operatorname{Cov}\left[\beta_{i p}, F_{r s}\right] \operatorname{Var}[F]_{r s t u}^{-1} \operatorname{Cov}\left[F_{t u}, \bar{u}_{j}\left(b^{\prime}\right)\right]
\end{aligned}
$$


with

$$
\begin{aligned}
\operatorname{Cov}\left[\bar{u}_{i}(b), u_{j}\left(\theta^{\prime}\right)\right] & =\int \operatorname{Cov}\left[u_{i}(a, b), u_{j}\left(\theta^{\prime}\right)\right] p(a) d a \\
\operatorname{Cov}\left[\bar{u}_{i}(b), \bar{u}_{j}\left(b^{\prime}\right)\right] & =\iint \operatorname{Cov}\left[u_{i}(a, b), u_{j}\left(a^{\prime}, b^{\prime}\right)\right] p(a) p\left(a^{\prime}\right) d a d a^{\prime}
\end{aligned}
$$

In Section 3.3 of the main article, this procedure is used to compute beliefs about the expected risk directly from our emulator for the risk.

\section{S1.4. Bayes Linear Uncertainty Analysis}

Frequently, when studying the behaviour of a set of properties of a real system $y(b)=\left\{y_{1}(b), \ldots, y_{n_{f}}(b)\right\}$ as a function of inputs $b$, we construct a function $f(a, b)$ which is designed to mimic its behaviour; in this context, we refer to $f$ as a simulator. The simulator $f$ is a function of the inputs $b$ at which we can measure system behaviour, but also of a set of simulator-specific inputs $a$ which do not affect system behaviour, but which must be specified in order to generate a prediction for $y$. A common approach (e.g. Goldstein and Rougier (2006), Craig et al. (1997)) to relating the simulator to the system is to assume the existence of an unknown 'best' input setting $a^{*}$ such that

$$
y_{i}(b)=f_{i}\left(a^{*}, b\right)+\delta_{i}(b)
$$

where $\delta_{i}(b)=\left\{\delta_{1}(b), \ldots, \delta_{n_{f}}(b)\right\}$ is the discrepancy between the simulator evaluated at $a^{*}$ and the system, assumed to be independent of $\left\{f, a^{*}\right\}$.

For computationally expensive simulators $f$, a common approach is to construct a surrogate emulator; we generate a set of runs $F$ on the simulator at known input settings, and then compute the adjusted moments $\mathrm{E}_{F}\left[f_{i}(a, b)\right]$ and $\operatorname{Cov}_{F}\left[f_{i}(a, b), f_{k}\left(a^{\prime}, b^{\prime}\right)\right]$ (as in Section S1.2), which we use to approximate the simulator at input settings where it has not been run. When coupled with a second-order uncertainty specification $\mathrm{E}\left[\delta_{i}(b)\right]$ and $\operatorname{Cov}\left[\delta_{i}(b), \delta_{k}\left(b^{\prime}\right)\right]$ for the discrepancy, this gives us a corresponding uncertainty specification for the system $y$ itself.

We can use a model constructed in this way to approximate the design calculation for the system. Using the notation of Section 2.1 from the main article, if we assume that our data $z_{j}$ are noise-corrupted measurements of the system, and we can divide the inputs $b$ into external and design inputs $\left\{w_{j}, d_{j}\right\}$ for each stage $j$, then

$$
z_{j p}=y_{p}\left(w_{j}, d_{j}\right)+\epsilon_{j p}
$$

where $\epsilon$ is assumed to be independent of $y$. Our beliefs about $z_{j}$ are then

$$
\begin{aligned}
& \mathrm{E}\left[z_{j p}\right]= \mathrm{E}\left[\hat{f}_{p}\left(w_{j}, d_{j}\right)\right]+\mathrm{E}\left[\delta_{p}\left(w_{j}, d_{j}\right)\right] \\
& \operatorname{Cov}\left[z_{j p}, z_{k q}\right]=\operatorname{Cov}\left[\hat{f}_{p}\left(w_{j}, d_{j}\right), \hat{f}_{q}\left(w_{k}, d_{k}\right)\right] \\
&+\operatorname{Cov}\left[\delta_{p}\left(w_{j}, d_{j}\right), \delta_{q}\left(w_{k}, d_{k}\right)\right]+\operatorname{Cov}\left[\epsilon_{j p}, \epsilon_{k q}\right]
\end{aligned}
$$


where $\hat{f}_{p}\left(w_{j}, d_{j}\right)=f_{p}\left(\left\{a_{1}^{*}, \ldots, a_{n_{a}}^{*}\right\},\left\{w_{j}, d_{j}\right\}\right)$, and the covariance of the data with the best input setting is

$$
\operatorname{Cov}\left[z_{j p}, a_{q}^{*}\right]=\operatorname{Cov}\left[\hat{f}_{p}\left(w_{j}, d_{j}\right), a_{q}^{*}\right]
$$

For carefully-selected emulator basis and covariance functions, we can compute the above moments algebraically from the expressions (S7) and (S8) (see, e.g. Craig et al. (2001), Goldstein and Rougier (2009)). If we are collecting the data $z_{j}$ with the intention of learning about the best input parameters $a^{*}$, then we can identify the model parameters with the best inputs $\left(q=a^{*}\right)$ and perform the approximate design procedure from Section 3 of the main article using this model specification. Wherever we must characterise the posterior distribution (1) (section 2.1.1 of the main article), we do so using our adjusted beliefs computed using the above second-order specification.

\section{S2. Design Algorithm}

In this section, we provide additional detail relating to the approximate sequential design algorithm presented in Section 3. In Section S2.1, we discuss the practical challenges relating to the fitting of an emulator as an approximation to the risk. Then, in Section S2.2, we consider the integration of the components of this model to obtain an approximation to the expected risk. In Section S2.3, we consider the implementation of the candidate design sampler from Section 3.4 of the main article. Finally, in Section S2.4, we consider how we might select an informative design for additional waves of the algorithm.

\section{S2.1. Fitting the Emulator}

In this Section, we consider practical aspects of fitting the model $r_{j}^{(i)}$ as an approximation to the risk $\rho_{j}$ at the $i^{\text {th }}$ wave.

\section{S2.1.1. Evaluating the Risk}

The risk evaluations that we use to fit the model $r_{j}^{(i)}$ are generated as in (10) (at the final stage, $j=n$ ) or (11) (all other stages, $j<n$ ); because of our uncertainty about $s_{j+1}^{(i)}$ (our approximation to the risk from an optimal design at stage $(j+1)$, defined in $(9))$, we cannot evaluate $(11)$ for $(j<n)$ directly. To fit our emulator then, we compute $\mathrm{E}\left[R_{j k}^{(i)}\right]$ and $\operatorname{Cov}\left[R_{j k}^{(i)}, R_{j l}^{(i)}\right]$, and we fit the emulator to the expected values, treating the covariance specification as the covariance of the measurement error.

When generating risk evaluations for stages $j<n$, we must compute moments of the expression (11); using only our second-order beliefs, this is not possible, and so we make a distributional specification for $s_{j+1}^{(i)}$ characterised by $\mathrm{E}\left[s_{(j+1) k}^{(i)}\right]$ 
and $\operatorname{Cov}\left[s_{(j+1) k}^{(i)}, s_{(j+1) l}^{(i)}\right]$ (computed as in Section S2.3) and characterise the first- and second-order moments of the $\left\{R_{j k}^{(i)}\right\}$ by sampling. Generally, we choose a multivariate Gaussian, as this is simple to characterise using a second-order specification; if we have specific beliefs about the higher-order moments of the risk, then we may choose a different distributional specification to reflect these.

\section{S2.1.2. Specifying the Prior}

Our first task when fitting an emulator of the form (7) is to choose a set of basis functions, and make a second-order prior specification for the regression coefficients and the residual process. Generally, a more accurate emulator can be fitted if we choose basis functions $g_{j p}^{(i)}$ that can describe as much of the global structure of the risk as possible. If the terminal risk $\rho_{j}^{\operatorname{trm}}$ is simple to evaluate, then using this (or a related approximation to (3)) as a basis function may be an effective choice.

Another important factor to consider when selecting the basis functions is our ability to compute their expectations with respect to $p\left(z_{j} \mid z_{[j-1]}, w_{[j]}, d_{[j]}\right)$ and $p\left(w_{j} \mid w_{[j-1]}, d_{[j]}\right)$ (as in equation (8), Section S2.2); the ability to integrate these functions algebraically will reduce the computational complexity of computing (8) later. If choosing to base the $g_{j p}^{(i)}$ on the terminal risk, then this can be achieved by replacing the inputs $z_{j}$ and $w_{j}$ with their conditional expectations $\mathrm{E}\left[z_{j} \mid z_{[j-1]}, w_{[j]}, d_{[j]}\right]$ and $\mathrm{E}\left[w_{j} \mid w_{[j-1]}, d_{[j]}\right]$, and then absorbing the effect on the output of variation around this mean level using the residual or nugget components. This strategy is adopted in both of the examples; see Sections S3 and $\mathrm{S} 4$.

Once the basis functions have been selected, we fix the prior moments of the $\beta_{j p}^{(i)}$. We do this by generating a sample of risks as outlined in Section S2.1.1, and using this to carry out an initial linear regression. The parameter estimates from this regression fit are used to fix $\mathrm{E}\left[\beta_{j p}^{(i)}\right]$ and $\operatorname{Cov}\left[\beta_{j p}^{(i)}, \beta_{j q}^{(i)}\right]$, and the residuals from the fitted surface are used to empirically fix the marginal variance $\operatorname{Var}\left[u_{j}^{(i)}\right]$ of the residual process.

\section{S2.1.3. Jointly Updating}

Using the prior specification made in Section S2.1.2, we jointly update the regression and residual components, as detailed in Section S1.2. As discussed in Section S1.2.2, the covariance function that we choose will generally depend on a number of correlation parameters; since a fully Bayesian treatment of these parameters would be too time-consuming, we instead fix these parameters using a leave-one-out cross validation procedure, and condition all subsequent calculations on this setting. 


\section{S2.1.4. Checking Inter-Wave Correspondence}

Using the procedure from Section 3, we have the option to perform multiple waves of analysis. At wave $i=1$, we emulate each of the risk functions for the first time; at subsequent waves $i=2,3, \ldots$ of the algorithm, however, we reemulate the same risk functions over sub-regions of the design input space. We hope that, as we focus on smaller portions of the design space, we will be able to more accurately capture risk behaviour using our models; at a minimum, however, we expect that the predictive error bars of the emulators fitted at wave $i$ should overlap with the predictive error bars of the emulators at waves $1, \ldots,(i-1)$. For each emulator fitted at later waves, this property can be checked to ensure that the behaviour of the procedure is as expected; failure to satisfy this criterion indicates that the fit of one or more of the emulators is a poor approximation to the risk.

\section{S2.2. Computed the Expected Risk}

Once the risk emulator $r_{j}^{(i)}$ has been fitted (Section S2.1), we integrate our model (7) to find our approximation $\bar{r}_{j}^{(i)}$ to the expected risk. At this step, we must be able to characterise the distributions $p\left(z_{j} \mid z_{[j-1]}, w_{[j]}, d_{[j]}\right)$ and $p\left(w_{j} \mid w_{[j-1]}, d_{[j]}\right)$, and compute the integrals of the basis and correlation functions with respect to these distributions. For general models $p\left(z_{j} \mid q, w_{j}, d_{j}\right)$ and prior specifications $p(q)$, these conditional distributions are complex objects; we may not be able to characterise them exactly, requiring us to rely on sampling algorithms or other numerical methods to investigate their characteristics.

For such complex distributions, we must numerically evaluate the integrals of basis or covariance functions, or adopt a strategy which approximately characterises the distributions in a way which allows us to perform the integrals directly. One possible strategy is to approximate using distributions characterised by the Bayes linear adjusted moments $\left\{\mathrm{E}_{z_{[j-1]}}\left[z_{j} \mid w_{[j]}, d_{[j]}\right], \operatorname{Var}_{z_{[j-1]}}\left[z_{j} \mid w_{[j]}, d_{[j]}\right]\right\}$ and $\left\{\mathrm{E}_{w_{[j-1]}}\left[w_{j} \mid d_{[j]}\right], \operatorname{Var}_{w_{[j-1]}}\left[w_{j} \mid d_{[j]}\right]\right\}$. If this, or any other, approximation strategy is adopted, its effect on our beliefs must be considered carefully.

\section{S2.3. Characterising the Optimal Design Space}

Trial design size The algorithm 3 is our general procedure for characterising the candidate design space. In order to use this procedure, we must choose a size $M_{j}^{(i)}$ for the trial design. Ideally, we would like to be able to determine the minimum sample size beyond which the characteristics of the sampled candidate designs do not change; however, there is no way to do this analytically, and it would be too computationally expensive to compare a large number of sizes $M_{j}^{(i)}$ empirically. Instead, then, we adopt a simple approach, where we generate samples of candidate designs $\tilde{d}_{j}$ and corresponding risks $\bar{r}_{j}^{(i)}+c_{j}\left(\tilde{d}_{j}\right)$ for a 
range of different values for $M_{j}^{(i)}$, and choose the design size which we judge to represent an appropriate trade-off between time to generate a sample, and stabilisation of sample characteristics.

Characterising the risk We cannot evaluate $s_{j}^{(i)}$ (equation (9)) exactly, since we do not know the optimal design setting $d_{j}^{*}$. Replacing the true optimal design with a candidate design $\tilde{d}_{j}$ sampled according to algorithm 3 , our beliefs about the risk within the candidate design space are

$$
\begin{aligned}
\mathrm{E}\left[s_{j}^{(i)}\right]= & \mathrm{E}\left[\mathrm{E}_{R_{j}^{(i)}}\left[\bar{r}_{j}^{(i)}\left[\tilde{d}_{j}\right]\right]+c_{j}\left(\tilde{d}_{j}\right)\right] \\
\operatorname{Cov}\left[s_{j}^{(i)},\left(s_{j}^{(i)}\right)^{\prime}\right]= & \mathrm{E}\left[\operatorname{Cov}_{R_{j}^{(i)}}\left[\bar{r}_{j}^{(i)}\left[\tilde{d}_{j}\right], \bar{r}_{j}^{(i)}\left[\tilde{d}_{j}\right]\right]\right] \\
& +\operatorname{Cov}\left[\mathrm{E}_{R_{j}^{(i)}}\left[\bar{r}_{j}^{(i)}\left[\tilde{d}_{j}\right]\right]+c_{j}\left(\tilde{d}_{j}\right), \mathrm{E}_{R_{j}^{(i)}}\left[\bar{r}_{j}^{(i)}\left[\tilde{d}_{j}\right]\right]+c_{j}\left(\tilde{d}_{j}\right)\right]
\end{aligned}
$$

where we have used the compact notations $s_{j}^{(i)}=s_{j}^{(i)}\left[z_{[j-1]}, w_{[j-1]}, d_{[j-1]}\right]$ and $\left(s_{j}^{(i)}\right)^{\prime}=s_{j}^{(i)}\left[z_{[j-1]}^{\prime}, w_{[j-1]}^{\prime}, d_{[j-1]}^{\prime}\right]$, we have suppressed the dependence on the inputs up to stage $(j-1)$ of all quantities on the right-hand side, and the outer expectations are taken with respect to $\tilde{d}_{j}$ by sampling design settings according to algorithm 3.

Sampling candidate designs according to algorithm 3 can be computationally demanding, and in large problems, repeatedly generating candidate designs $\tilde{d}_{j}$ for each input setting may be computationally infeasible. In such problems, we simplify the approach further by characterising using a single candidate design; we simply set $\mathrm{E}\left[s_{j}^{(i)}\right]$ to be the expectation of $\bar{r}_{j}^{(i)}$ at the sampled point, and the first term of (S9) is set to be the covariance between $\bar{r}_{j}^{(i)}$ values at the two different input settings. For the second term in equation (S9), we approximate by sampling the variance of the expectation within the candidate design space at an handful of different input settings, and then fixing the variance of the expectation for any input setting to the average of these values; the covariances between pairs of expectations at different input settings are fixed to zero.

Simple characterisation of design spaces As written, the procedure 3 for sampling candidate minima is recursive; we must be able to generate a set of trial designs inside the space $\mathcal{D}_{j}^{(i-1)}$ in order to sample from $\mathcal{D}_{j}^{(i)}$. For problems where we carry out multiple waves, it is likely that a recursive implementation will be infeasible. Instead, we may choose to approximate the candidate design space by characterising each space $\mathcal{D}_{j}^{(i)}$ in terms of simple limits. Approximating the spaces in this way will capture the ranges of design values which we have not yet ruled out, but will ignore any dependencies between the components of $d_{j}$ induced by the shape of the risk emulator. 


\section{S2.4. Designing for Future Waves}

At the first wave of the algorithm, we select the design inputs used to generate the data $R_{j}^{(1)}$ in a space-filling way (for example, a Latin hypercube); this is a common approach to achieving good coverage of the input space when fitting emulators (see e.g. Vernon et al. (2010)). At later waves $i=2,3, \ldots$, we re-fit emulators in sub-regions of the design space in order to build up a more accurate picture of risk behaviour; we select the design inputs used for the emulator fits at these waves so that they focus only on design input regions which our emulators at wave $i$ indicate may be interesting.

At wave $i>1$, we choose design inputs $d_{j}$ for the emulator fit which:

- lie in the candidate design space $\mathcal{D}_{j}^{(i-1)}$ (characterised as in algorithm 3), and;

- satisfy the following criterion for $j>1, k=1, \ldots,(i-1)$

$$
\frac{\mathrm{E}_{R_{j}^{(i)}}\left[r_{j}^{(i)}\left[z_{[j-1]}, w_{[j-1]}, d_{[j]}\right]\right]+c_{j}\left(d_{j}\right)-\rho_{j-1}^{\operatorname{trm}}\left[z_{[j-1]}, w_{[j-1]}, d_{[j-1]}\right]}{\operatorname{Var}_{R_{j}^{(i)}}\left[r_{j}^{(i)}\left[z_{[j-1]}, w_{[j-1]}, d_{[j]}\right]\right] 1 / 2} \leq 3
$$

By the three-sigma rule of Pukelsheim (1994) (for continuous, unimodal distributions), the criterion (S10) corresponds to a specification that we only select design inputs for which there is a greater than $5 \%$ chance under our current beliefs that we will continue sampling at least as far as stage $j$.

\section{S3. Example: Linear Model}

In this section, we provide additional detail relating to the linear model example presented in Section 4 of the main article.

\section{S3.1. First Wave: Details}

\section{S3.1.1. Stage 2: Emulating the risk}

We begin the first wave by fitting $r_{2}^{(1)}$ as an approximation to $\rho_{2}$. Following the procedure outlined in Section S2.1, we begin by selecting basis functions for this emulator; at this stage, the risk $\rho_{2}=\rho_{2}^{\text {trm }}$, and so we select the terminal risk as the basis of our approximation. We fix $g_{21}^{(1)}=1$ and $g_{22}^{(1)}=\rho_{2}^{\operatorname{trm}}\left[\tilde{z}_{[2]}, d_{[2]}\right]$, where $\tilde{z}_{[2]}=\left\{z_{[1]}, \mathrm{E}\left[z_{2} \mid z_{[1]}, d_{[2]}\right]\right\}$ is the set of data inputs with $z_{2}$ substituted for its conditional expectation. For the covariance function, we choose a separable form, as follows

$$
\operatorname{Cov}\left[u_{2}^{(1)}\left(z_{[2]}, d_{[2]}\right), u_{2}^{(1)}\left(z_{[2]}^{\prime}, d_{[2]}^{\prime}\right)\right]=V_{2}^{(1)} \prod_{j=1}^{2}\left[k_{\text {se }}\left(z_{j}, z_{j}^{\prime} \mid \lambda_{z}\right) k_{\mathrm{se}}\left(d_{j}, d_{j}^{\prime} \mid \lambda_{d}\right)\right]
$$


where $k_{\mathrm{se}}$ is a squared exponential (SE) correlation function (see Appendix A). Note that we specify the same correlation lengths for each input at both stages. These choices of basis and covariance function ensure that we will be able to compute the moments of (8) algebraically. We carry out out initial regression using 200 risk evaluations, fixing the prior specification for the regression components and the marginal variance $V_{2}^{(1)}$ of the residual process. We then jointly update the regression and residual components using 300 risk evaluations, fixing the correlation lengths $\left\{\lambda_{z}, \lambda_{d}\right\}$ using leave-one-out cross validation (where we test 2000 candidate settings, chosen according to a Latin hypercube, and choose the best of these). Finally, we check our fitted emulator against a new sample of 100 risks; none of these lie outside predictive three-standard deviation error bars.

\section{S3.1.2. Stage 2: Computing the expected risk}

Once the emulator has been fitted, we characterise our approximation $\bar{r}_{2}^{(1)}$ to the expected risk $\bar{\rho}_{2}$ at this stage (equation (8), Section S1.3). Because of the simplicity of the problem, the distribution $p\left(z_{2} \mid z_{[1]}, d_{[2]}\right)$ is a Gaussian. The basis function was designed not to depend on $z_{2}$ so we have that $\bar{g}_{22}^{(1)}=g_{22}^{(1)}$. For the residual process, to compute the moments from Section S1.3, we compute integrals of the covariance function. Integrating in the first argument, we have

$$
\begin{aligned}
\operatorname{Cov}\left[\bar{u}_{2}^{(1)}\left(z_{[1]}, d_{[2]}\right), u_{2}^{(1)}\left(z_{[2]}^{\prime}, d_{[2]}^{\prime}\right)\right]= & V_{2}^{(1)} k_{\text {se }}\left(z_{1}, z_{1}^{\prime} \mid \lambda_{z}\right) k_{\text {se }}\left(d_{1}, d_{1}^{\prime} \mid \lambda_{d}\right) \\
& \times \bar{k}_{\text {se }}\left(z_{1}^{\prime} \mid \lambda_{z}\right) k_{\text {se }}\left(d_{2}, d_{2}^{\prime} \mid \lambda_{d}\right)
\end{aligned}
$$

where $\bar{k}_{\text {se }}$ is the SE correlation function integrated once with respect to $p\left(z_{2} \mid z_{[1]}, d_{[2]}\right)$ (see Appendix A.1). Integrating again, we have

$$
\begin{aligned}
\operatorname{Cov}\left[\bar{u}_{2}^{(1)}\left(z_{[1]}, d_{[2]}\right), \bar{u}_{2}^{(1)}\left(z_{[1]}^{\prime}, d_{[2]}^{\prime}\right)\right]= & V_{2}^{(1)} k_{\text {se }}\left(z_{1}, z_{1}^{\prime} \mid \lambda_{z}\right) k_{\text {se }}\left(d_{1}, d_{1}^{\prime} \mid \lambda_{d}\right) \\
& \times \overline{\bar{k}}_{\text {se }}\left(\lambda_{z}\right) k_{\text {se }}\left(d_{2}, d_{2}^{\prime} \mid \lambda_{d}\right)
\end{aligned}
$$

where $\overline{\bar{k}}_{\mathrm{se}}($.$) is the SE function integrated in both arguments (see Appendix$ A.1).

\section{S3.1.3. Stage 2: Characterising the candidate design space}

Finally at this stage, we assess the additional variance contribution to $s_{2}^{(1)}$ that we include to represent our uncertainty about the location of the optimal design (Section S2.3). We set the design size for the candidate design sampling algorithm to $M_{2}^{(1)}=200$, and we generate 20 candidate designs and corresponding risks at each of 10 input settings $\left\{z_{[1]}, d_{[1]}\right\}$ using algorithm 3 . As outlined in section S2.3, we fix the second component of (S9) to $\left(7.8 \times 10^{-4}\right)^{2}$ and assume that this is constant across the input domain. 


\section{S3.1.4. Stage 1}

We now fit the emulator $r_{1}^{(1)}$ which approximates the risk at the first stage. We follow the procedure in Section S2.1; our basis functions are $g_{11}^{(1)}=1$ and $g_{12}^{(1)}=\rho_{1}^{\operatorname{trm}}\left[\tilde{z}_{[1]}, d_{[1]}\right]$, and our covariance function again has a separable squared exponential form

$$
\operatorname{Cov}\left[u_{1}^{(1)}\left(z_{[1]}, d_{[1]}\right), u_{1}^{(1)}\left(z_{[1]}^{\prime}, d_{[1]}^{\prime}\right)\right]=V_{1}^{(1)} k_{\mathrm{se}}\left(z_{1}, z_{1}^{\prime} \mid \lambda_{z}\right) k_{\mathrm{se}}\left(d_{1}, d_{1}^{\prime} \mid \lambda_{d}\right)
$$

For this emulator, we carry out our initial regression using 200 risk evaluations, and we perform our joint update and cross-validation using 500 risk points (with 2000 candidate correlation parameter settings for the cross-validation). We check the fitted emulator by predicting an additional set of 100 risk points, and find that $1 \%$ lie outside predictive three-standard deviation error bars. The integrals of the covariance function with respect to $p\left(z_{1} \mid d_{[1]}\right)$ correspond to those at the second wave.

\section{S3.2. Second Wave: Details}

Stage 2 We judge that the basis and covariance functions that were used at the first wave performed well, and so we choose to make the same specifications for all stages at this wave. To fit the emulator $r_{2}^{(2)}$, we use 200 risk points for the initial regression, 300 for the joint update and 100 for model checking. Again, 2000 candidate correlation settings are tested in the cross-validation. Again, none of the validation data lie outside of the three-standard deviation error bars.

After fitting, we compute the additional variance contribution to $s_{2}^{(2)}$ (Section S2.3). Again, we set the design size to $M_{2}^{(2)}=200$, and we generate 20 candidate designs and corresponding risks at each of 10 input locations using algorithm 3 . We fix the second component of equation (S9) to $\left(6.7 \times 10^{-4}\right)^{2}$, the average of these values, and assume that this is constant across the input domain.

Stage 1 To fit this emulator $\left(r_{1}^{(2)}\right)$, we use 200 risk evaluations for the initial regression, 200 for the joint update (with 2000 correlation settings tested in the cross-validation) and 100 for model checking. All of the validation points are found to lie within three-standard deviation error bars.

\section{S4. Example: Airborne Sensing Problem}

In this section, we provide additional detail relating to the atmospheric dispersion example presented in Section 5 of the main article. 


\section{S4.1. Prior Specification and Posterior Risk}

We make a prior second-order specification for all of the components of the model (15) introduced in Section 5.2. Previous treatments of this problem have opted for more complex prior specifications, e.g. Hirst et al. (2012) allowed for an unknown number of sources at unknown locations, and carried out their inference using a reversible jump MCMC scheme; in our treatment, we opt instead for this simple, second-order specification, as it allows us to characterise the reduction in uncertainty achieved by observing particular data sets, while ensuring the tractability of the design calculations. The prior specification consists of the components $\mathrm{E}\left[q_{k}\right]$ and $\operatorname{Cov}\left[q_{k}, q_{l}\right]$ for each of the model parameters $q_{k}=\psi_{k}$, and $\operatorname{Var}\left[\epsilon_{j p}\right]$ for the noise components $\epsilon_{j p}$ (we assume that $\operatorname{Cov}\left[\epsilon_{j p}, \epsilon_{k q}\right]=0$ for $j p \neq k q$ ).

For the chosen loss function, the terminal risk in this problem is given in equation (16) (Section 5.2); in general, this depends on the posterior covariances between pairs of model parameters. Under our second-order specification, then, we simply characterise this risk using the adjusted covariance $\operatorname{Cov}_{z_{[j]}}\left[q_{k}, q_{l} \mid w_{[j]}, d_{[j]}\right]$.

\section{S4.2. Emulator Fit: Details}

Basis and covariance functions We make the same choices for the basis and covariance functions at all three stages of the problem, at both waves. We specify that $g_{j 1}^{(i)}=1$ for $j=1,2,3$, that at stage $n$

$$
g_{n 2}^{(i)}\left(z_{[n]}, w_{[n]}, d_{[n]}\right)=\rho_{n}^{\operatorname{trm}}\left[\tilde{z}_{[n]}, \tilde{w}_{[n]}, d_{[n]}\right]
$$

and that at stages $j<n$

$$
\begin{aligned}
& g_{j 2}^{(i)}\left(z_{[j]}, w_{[j]}, d_{[j]}\right)= \\
& \quad \min \left[\rho_{j}^{\operatorname{trm}}\left[\tilde{z}_{[j]}, \tilde{w}_{[j]}, d_{[j]}\right], \mathrm{E}_{R_{j+1}^{(1)}}\left[\bar{r}_{j+1}^{(i)}\left[\tilde{z}_{[j]}, \tilde{w}_{[j]}, \tilde{d}_{[j+1]}\right]\right]+c_{j+1}\left(\xi_{j+1}\right)\right]
\end{aligned}
$$

where $\tilde{z}_{[j]}=\left\{z_{[j-1]}, \mathrm{E}_{z_{[j-1]}}\left[z_{j} \mid \tilde{w}_{[j]}, d_{[j]}\right]\right\}, \tilde{w}_{[j]}=\left\{w_{[j-1]}, \mathrm{E}\left[w_{j}\right]\right\}$, and $\tilde{d}_{[j+1]}=$ $\left\{d_{[j]}, \xi_{j+1}\right\}$, where $\xi_{j+1}$ is a pre-specified design setting, selected in a region of the design space which, based on initial data exploration, we believe may be near-optimal for a wide range of risk input settings at the previous stages.

For the covariance function, we again choose a separable squared exponential form at all stages

$$
\begin{aligned}
\operatorname{Cov}\left[u_{j}^{(i)}\left(z_{[j]}, w_{[j]}, d_{[j]}\right), u_{j}^{(i)}\left(z_{[j]}^{\prime}, w_{[j]}^{\prime}, d_{[j]}^{\prime}\right)\right]= \\
V_{j}^{(i)} \prod_{p=1}^{j}\left[\left[\prod_{q=1}^{2} k_{\mathrm{se}}\left(w_{p q}, w_{p q} \mid \lambda_{w_{q}}\right)\right]\left[\prod_{q=1}^{5} k_{\mathrm{se}}\left(d_{p q}, d_{p q} \mid \lambda_{d_{q}}\right)\right]\right]
\end{aligned}
$$

where $k_{\text {se }}(., . \mid \lambda)$ is defined in Appendix A, and we specify that the correlation parameters $\lambda_{w_{q}}$ and $\lambda_{d_{q}}$ corresponding to particular external and design inputs are the same across all stages $j$. When fitting an emulator, the parameters 
$\left\{\lambda_{w_{1}}, \lambda_{w_{2}}, \lambda_{d_{1}}, \ldots, \lambda_{d_{5}}\right\}$ are fixed using cross-validation (see Section S1.2.2).

In order to characterise $\bar{r}_{j}^{(i)}$, we must compute integrals of the basis and covariance functions (Section 3.3 of the main article). Because of the way the basis functions have been selected, we simply have that $\bar{g}_{j}^{(i)}=g_{j}^{(i)}$ for all $i, j$. For the covariance function, integrating in the first argument, we have that

$$
\begin{aligned}
& \operatorname{Cov}\left[\bar{u}_{j}^{(i)}\left(z_{[j-1]}, w_{[j-1]}, d_{[j]}\right), u_{j}^{(i)}\left(z_{[j]}^{\prime}, w_{[j]}^{\prime}, d_{[j]}^{\prime}\right)\right]= \\
& V_{j}^{(i)}\left[\prod_{q=1}^{2} \bar{k}_{\mathrm{se}}\left(w_{j q}^{\prime} \mid \lambda_{w_{q}}\right)\left[\prod_{p=1}^{j-1} k_{\mathrm{se}}\left(w_{p q}, w_{p q} \mid \lambda_{w_{q}}\right)\right]\right] \\
& \times\left[\prod_{p=1}^{j} \prod_{q=1}^{5} k_{\mathrm{se}}\left(d_{p q}, d_{p q} \mid \lambda_{d_{q}}\right)\right]
\end{aligned}
$$

and integrating again in the second argument, we have that

$$
\begin{array}{r}
\operatorname{Cov}\left[\bar{u}_{j}^{(i)}\left(z_{[j-1]}, w_{[j-1]}, d_{[j]}\right), \bar{u}_{j}^{(i)}\left(z_{[j-1]}^{\prime}, w_{[j-1]}^{\prime}, d_{[j]}^{\prime}\right)\right]= \\
V_{j}^{(i)}\left[\prod_{q=1}^{2} \overline{\bar{k}}_{\mathrm{se}}\left(\lambda_{w_{q}}\right)\left[\prod_{p=1}^{j-1} k_{\mathrm{se}}\left(w_{p q}, w_{p q} \mid \lambda_{w_{q}}\right)\right]\right] \\
\times\left[\prod_{p=1}^{j} \prod_{q=1}^{5} k_{\mathrm{se}}\left(d_{p q}, d_{p q} \mid \lambda_{d_{q}}\right)\right]
\end{array}
$$

where $\bar{k}_{\text {se }}(. \mid \lambda)$ and $\overline{\bar{k}}_{\text {se }}(\lambda)$ are computed as in Appendix A.2

\section{Appendix A: Squared Exponential Correlation Function}

In both of the examples (Sections 4 and 5 of the main article), we use squared exponential correlation functions for our risk emulators. This has the following form for general scalar inputs $\left\{x, x^{\prime}\right\}$

$$
k_{\text {se }}\left(x, x^{\prime} \mid \lambda\right)=\exp \left[-\frac{\lambda}{2}\left(x-x^{\prime}\right)^{2}\right]
$$

where $\lambda$ is a correlation parameter which governs the rate at which the correlation between function outputs at $x$ and $x^{\prime}$ decays to zero as the squared distance between the inputs increases. Correlation functions for multi-input functions are constructed as products of correlation functions for the individual inputs; see Sections S3.1 and S4.2. When emulating, the parameters $\lambda$ are fixed through cross validation; see Section S1.2.2.

The SE correlation function is chosen for its simplicity, and because it is simple to integrate with respect to both Gaussian and uniform distributions. In Section A.1, we compute integrals of a single function with respect to a Gaussian distribution (the approach taken in Section S3.1) and in Section A.2, we compute integrals with respect to a uniform distribution (the approach taken in Section S4.2). 


\section{A.1. Integrals: Gaussian Case}

Suppose that $x \sim \mathcal{N}(\mu, v)$; then, the integral of $k_{\mathrm{se}}$ in its first argument is

$$
\begin{aligned}
\bar{k}_{\text {se }}\left(x^{\prime} \mid \lambda\right) & =\int_{-\infty}^{\infty} k_{\text {se }}\left(x, x^{\prime} \mid \lambda\right) p(x) d x \\
& =\sqrt{\frac{2 \pi}{\lambda}} \int_{-\infty}^{\infty}\left[\sqrt{\frac{\lambda}{2 \pi}} \exp \left[-\frac{\lambda}{2}\left(x^{\prime}-x\right)^{2}\right] \frac{1}{\sqrt{2 \pi v}} \exp \left[-\frac{1}{2 v}(x-\mu)^{2}\right]\right] d x \\
& =\sqrt{\frac{2 \pi}{\lambda}}\left[\frac{1}{\sqrt{2 \pi \hat{v}}} \exp \left[-\frac{1}{2 \hat{v}}\left(x^{\prime}-\mu\right)^{2}\right]\right]
\end{aligned}
$$

using standard results for the Gaussian distribution, where $\hat{v}=v+\frac{1}{\lambda}$. If $x^{\prime} \sim$ $\mathcal{N}\left(\mu^{\prime}, v^{\prime}\right)$, then the integral of the SE correlation function in both arguments is

$$
\begin{aligned}
\overline{\bar{k}}_{\mathrm{se}}(\lambda) & =\int_{-\infty}^{\infty} \int_{-\infty}^{\infty} k_{\mathrm{se}}\left(x, x^{\prime} \mid \lambda\right) p(x) p\left(x^{\prime}\right) d x d x^{\prime} \\
& =\int_{-\infty}^{\infty} \bar{k}_{\mathrm{se}}\left(x^{\prime} \mid \lambda\right) p\left(x^{\prime}\right) d x^{\prime} \\
& =\sqrt{\frac{2 \pi}{\lambda}}\left[\frac{1}{\sqrt{2 \pi \hat{\hat{v}}}} \exp \left[-\frac{1}{2 \hat{\hat{v}}}\left(\mu-\mu^{\prime}\right)^{2}\right]\right]
\end{aligned}
$$

where $\hat{\hat{v}}=v+v^{\prime}+\frac{1}{\lambda}$.

\section{A.2. Integrals: Uniform Case}

In the case where $x \sim \mathcal{U}(l, u)$ has a uniform distribution on $[l, u]$, then the integral of $k_{\mathrm{se}}$ in its first argument is

$$
\begin{aligned}
\bar{k}_{\mathrm{se}}\left(x^{\prime} \mid \lambda\right) & =\int_{-\infty}^{\infty} k_{\mathrm{se}}\left(x, x^{\prime} \mid \lambda\right) p(x) d x \\
& =\int_{l}^{u} \exp \left[-\frac{\lambda}{2}\left(x^{\prime}-x\right)^{2}\right] \frac{1}{(u-l)} d x \\
& =\frac{1}{(u-l)} \sqrt{\frac{2 \pi}{\lambda}}\left[\Phi\left(\sqrt{\lambda}\left(u-x^{\prime}\right)\right)-\Phi\left(\sqrt{\lambda}\left(l-x^{\prime}\right)\right)\right]
\end{aligned}
$$

where $\Phi$ is the CDF for a standard Gaussian distribution $\mathcal{N}(0,1)$. Assuming that $x^{\prime} \sim \mathcal{U}\left(l^{\prime}, u^{\prime}\right)$, the integral in both arguments is

$$
\begin{aligned}
\overline{\bar{k}}_{\mathrm{se}}(\lambda)=\int_{-\infty}^{\infty} \bar{k}_{\mathrm{se}}\left(x^{\prime} \mid \lambda\right) p\left(x^{\prime}\right) d x^{\prime} & \\
=\frac{1}{(u-l)\left(u^{\prime}-l^{\prime}\right)} \sqrt{\frac{2 \pi}{\lambda}} & {\left[\left(\bar{\Phi}\left(\sqrt{\lambda}\left(u-u^{\prime}\right)\right)-\bar{\Phi}\left(\sqrt{\lambda}\left(u-l^{\prime}\right)\right)\right)\right.} \\
& \left.-\left(\bar{\Phi}\left(\sqrt{\lambda}\left(l-u^{\prime}\right)\right)-\bar{\Phi}\left(\sqrt{\lambda}\left(l-l^{\prime}\right)\right)\right)\right]
\end{aligned}
$$


where

$$
\bar{\Phi}(\eta)=\frac{-1}{\sqrt{\lambda}}[\eta \Phi(\eta)+\phi(\eta)]
$$

and $\phi$ is the PDF of a standard Gaussian distribution $\mathcal{N}(0,1)$.

\section{References}

P.S. Craig, M. Goldstein, J.C. Rougier, and A.H. Seheult [2001]. "Bayesian Forecasting for Complex Systems Using Computer Simulators." Journal of the American Statistical Association, 96:717-729.

P.S. Craig, M. Goldstein, A.H. Seheult, and J.A. Smith [1997]. "Pressure Matching for Hydrocarbon Reservoirs: A Case Study in the Use of Bayes Linear Strategies for Large Computer Experiments." In C. Gatsonis, J.S. Hodges, R.E. Kass, R. McCulloch, P. Rossi, and N.D. Singpurwalla, editors, "Case Studies in Bayesian Statistics," volume 3, pages 36-93. New York: SpringerVerlag.

M. Goldstein and J. Rougier [2004]. "Probabilistic Formulations for Transferring Inferences from Mathematical Models to Physical Systems." SIAM Journal on Scientific Computing, 26:467-487. .

M. Goldstein and J. Rougier [2006]. "Bayes Linear Calibrated Prediction for Complex Systems." Journal of the American Statistical Association, 475:1132-1143. .

M. Goldstein and J. Rougier [2009]. "Reified Bayesian Modelling and Inference for Physical Systems." Journal of Statistical Planning and Inference, 139:1221-1239. .

M. Goldstein and D. Wooff [2007]. Bayes Linear Statistics: Theory and Methods. Wiley.

B. Hirst, P. Jonathan, F. Gonzalez del Cueto, D. Randell, and O. Kosut [2012]. "Locating and Quantifying Gas Emission Sources Using Remotely Obtained Concentration Data." Atmospheric Environment, 45:141-158.

M.C. Kennedy and A. O'Hagan [2001]. "Bayesian Calibration of Computer Models." Journal of the Royal Statistical Society, Series B (Statistical Methodology), 63:425-464.

A. O'Hagan [1991]. "Bayes-Hermite Quadrature." Journal of Statistical Planning and Inference, 29:245-260.

F. Pukelsheim [1994]. "The Three Sigma Rule." The American Statistician, 48:88-91.

C.E. Rasmussen and Z. Ghahramani [2002]. "Bayesian Monte Carlo." In "Advances in Neural Information Processing Systems," MIT Press.

C.E. Rasmussen and C.K.I. Williams [2006]. Gaussian Processes for Machine Learning. The MIT Press.

I. Vernon, M. Goldstein, and R.G. Bower [2010]. "Galaxy Formation: a Bayesian Uncertainty Analysis." Bayesian Analysis, 5:619-669. . 